\title{
Perceptually-based Gamut Extension Algorithm for Emerging Wide Color Gamut Display and Projection Technologies
}

\section{Syed Waqas Zamir}

Universitat Pompeu Fabra, Barcelona, Spain, waqas.zamir@upf.edu

\section{Javier Vazquez-Corral}

Universitat Pompeu Fabra, Barcelona, Spain, javier.vazquez@upf.edu

\section{Marcelo Bertalmío}

Universitat Pompeu Fabra, Barcelona, Spain, marcelo.bertalmio@upf.edu

\section{Written for presentation at the}

\section{SMPTE 2016 Annual Technical Conference \& Exhibition}

\begin{abstract}
While wide color gamut (WCG) capabilities are a key element of emerging display and projection technologies, at present most image content is recorded using standards such as DCl-P3 for cinema or BT.709 for TV that have a reduced color gamut. Therefore, there is a need for gamut extension methods that process regular content and allow to appreciate the full color potential of new displays, improving user experience. We present a gamut extension algorithm that is based on visual perception models and explicitly takes into account low chromatic colors such as skin tones. It produces results that look natural, are free of artifacts of any kind, and outperform the state of the art. The method is fast, allowing for operation interaction if needed.
\end{abstract}

Keywords. Wide color gamut, gamut mapping, color management, gamut extension algorithms.

The authors are solely responsible for the content of this technical presentation. The technical presentation does not necessarily reflect the
official position of the Society of Motion Picture and Television Engineers (SMPTE), and its printing and distribution does not constitute an
endorsement of views which may be expressed. This technical presentation is subject to a formal peer-review process by the SMPTE Board
of Editors, upon completion of the conference. Citation of this work should state that it is a SMPTE meeting paper. EXAMPLE: Author's Last
Name, Initials. 2011. Title of Presentation, Meeting name and location.: SMPTE. For information about securing permission to reprint or
reproduce a technical presentation, please contact SMPTE at jwelch@smpte.org or 914-761-1100 (3 Barker Ave., White Plains, NY 10601). 


\section{Introduction}

The normal human eye contains three types of cone cells that respond to incident light in order to produce the sensation of color. Numerically, it is possible to match a wide range of colors by mixing three properly chosen color primaries ${ }^{1}$, resulting in a three-dimensional space where each point represents a color. However, when describing colors, it is a common practice to decouple luminance from the chromatic content referred as chromaticity. Figure 1 shows the standard CIE xy chromaticity diagram, where the tongue-shaped region corresponds to the chromaticities of all the colors that our eyes are capable of perceiving. The possible range of colors that a given display device can render is referred as 'color gamut'. Practically all commercially available displays have a triangle-shaped gamut in the CIE xy chromaticity diagram due to the use of three color primaries ${ }^{2}$. Therefore, they are unable to reproduce many colors that we can see.

Some studies ${ }^{3,4}$ tried investigating how much color gamut do displays really need. M. R. Pointer ${ }^{4}$ analyzed a set of frequently occurring real surface colors and derived what is commonly known as "Pointer's gamut", shown in figure 1. In order to ensure a consistent movie presentation with old or standard display devices, at the post-production stage, colorists reproduce colors of movies according to standard gamuts (DCl-P35 for cinema and BT. $709^{6}$ for broadcast) by using threedimensional look up tables and by performing intensive manual correction in a shot-by-shot, object-by-object basis. Although both the DCI-P3 and BT.709 gamuts cover a reasonable amount of Pointer's gamut, many interesting real world colors fall outside these standard gamuts. In 2012, the International Telecommunication Union-Radiocommunication (ITU-R) recommended a new standard gamut BT.2020 7 for the next generation ultra-high definition TV that encompasses existing video standards ${ }^{5,6}$ and covers $99.9 \%$ of Pointer's gamut. All of the aforementioned gamuts are shown in figure 1. Unlike standard displays, new laser projectors ${ }^{8,9}$ due to their monochromatic primaries with high color purity ${ }^{10}$, are capable to cover the very wide BT.2020 gamut, reproducing nearly every color found in nature and providing the audience with a visual experience close to real life. But if the inputs are movies with either DCI-P3 gamut or BT.709 gamut, as virtually all professional movies currently are, the full color potential of these new displays can not be realized. Hence, there is a need to develop automatic gamut extension (GE) techniques.

To perform GE, one could possibly think of stretching out (linearly or non-linearly) ${ }^{11}$ the input signal to a wider gamut in order to have a boost in the saturation of colors. This way of addressing the GE problem is simple but prone to several issues. Chief among these problems is that the GE procedure may alter the artistic intent of the content's creator. Also, memory colors such as the blue of the sky and the green of the grass may look unrealistic after the application of GE, requiring a special treatment ${ }^{12,13}$. Another challenge is to preserve skin tones ${ }^{12,14}$, always a key issue in movie postproduction, but many gamut extension algorithms (GEAs) ${ }^{15,16,17}$ fail to do so, as reported by Morovic ${ }^{18}$. Therefore, the fundamental goal of GE should be to reproduce input material in accordance with the color characteristics of a display device such that the appearance of the reproduction matches closely to the original material, perceptually. 


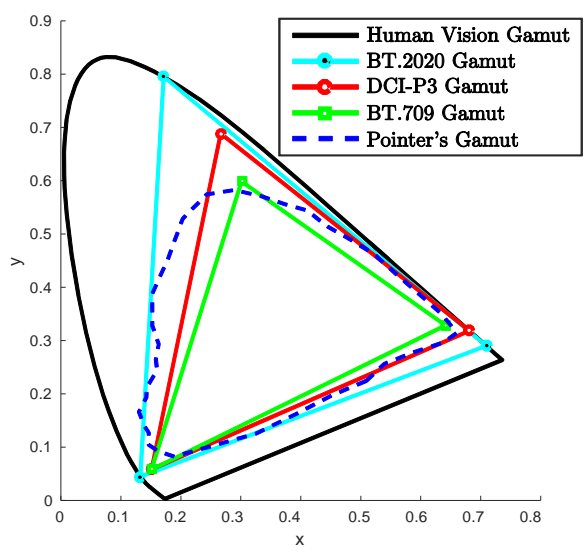

Figure 1. Gamuts on CIE xy chromaticity diagram.

\section{Perceptually-based Gamut Extension}

In the HSV color space, the GE goal can be formulated as increasing saturation while keeping the hue and value channels constant. Bertalmío et al. $^{19}$ propose a perceptual function that increases contrast while being monotonically increasing, meaning that it is able to increase the contrast but does not decrease the image values. Their algorithm ${ }^{19}$ complies with some global and local properties of the human visual system. In order to map colors from a small source gamut to a larger destination gamut, in this paper we present a GEA adapting the work of Bertalmío et al. ${ }^{19}$ and introducing some key modifications to it by taking into account that some colors require a special treatment. The following iterative scheme adapted from the method ${ }^{19}$ progressively increases the saturation of the input image in order to extend its gamut:

$$
S^{k+1}(x)=\frac{S^{k}(x)+\Delta t\left(S_{0}(x)\left(\beta+\tau\left(S_{0}(x)\right) V_{0}(x)^{2}\right)+\frac{\gamma}{2} R_{S^{k}}(x)\right)}{1+\Delta t\left(\beta+\tau\left(S_{0}(x)\right) V_{0}(x)^{2}\right)}
$$

where $S(x)$ represents the saturation level at pixel location $x$ in the input image. $\beta$ is a positive weight that controls the departure of the output image's saturation from the original image's saturation $S_{0} . V_{0}$ is the value component (HSV color space) of the original image, $\Delta t$ is the time step and $k \in \mathbb{N}$ denotes the iteration number. In gamut extension, certain colors require a special treatment in order to look natural and pleasant such as skin tones, less saturated natural objects, neutral colors and some particular memory colors. To incorporate such a functionality into the proposed GEA, the function $\tau(\cdot)$, shown in figure 2, takes as an argument the saturation of the original image and associates weights with the pixels of the image. These weights indicate the amount of special treatment we provide to colors of the input image. For example, the low saturated colors of the input image that require little to no extension are given higher weights $\tau(\cdot)$, whereas low weights are attached to extend normally the high saturated (artificial objects) colors. With this we can extend the gamut of the input image in a controlled manner treating objects of low saturation and high saturation differently. And to compute these weights, we make use of the 


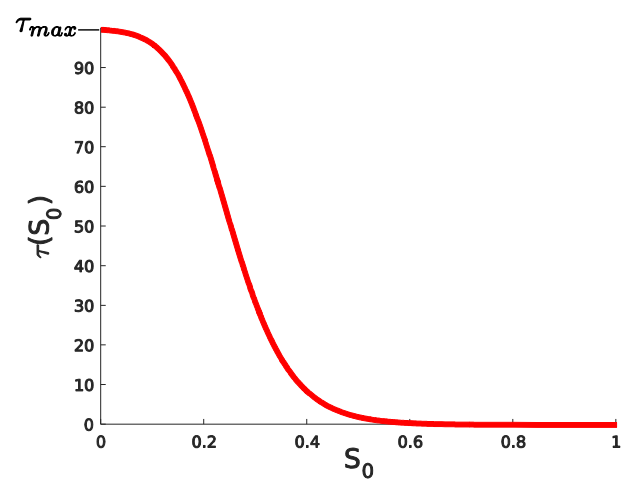

Figure 2. Logistic function.

following function derived from the generalised logistic function ${ }^{20}$ :

$$
\tau\left(S_{0}(x)\right)=\tau_{\max }\left(1-\frac{1}{1+0.2 e^{-3 S_{0}(x)^{2}}}\right)
$$

where $\tau_{\max }$ is a positive constant. The values used in Eq. (2) have been chosen based on tests we performed on several images with different color characteristics.

In Eq. (1) the initial condition is $S^{k=0}(x)=S_{0}(x)$, and $\gamma$ is a positive constant that allows us to make increments in the contrast function $R_{S^{k}}(x)$ in order to expand the color gamut:

$$
R_{S^{k}}(x)=\sum_{y \in \mathfrak{I}} w(x, y)\left[f\left(\frac{S^{k}(x)}{S^{k}(y)}\right) \operatorname{sign}^{+}\left(S^{k}(y)-S^{k}(x)\right)+\operatorname{sign}^{-}\left(S^{k}(y)-S^{k}(x)\right)\right]
$$

since Eq. (3) involves ratios, we normalize the dynamic range of $S$ in $(0,1]$ so as to avoid division by zero. This, for example, could be done on a 8-bit-per-channel image just by adding 1 to each pixel and diving by 256. The scaling function $f^{19}$ is strictly increasing such that $f(r) \geq r$ for all $r \in$ $(0,1], w(x, y)$ is a normalized Gaussian kernel of standard deviation $\sigma$, and the functions $\operatorname{sign}^{+}()$ and $\operatorname{sign}^{-}()$are, respectively, defined as:

$$
\begin{aligned}
& \operatorname{sign}^{+}(\xi)= \begin{cases}1, & \text { if } \xi>0 \\
\frac{1}{2}, & \text { if } \xi=0 \\
1 & \text { if } \xi<0\end{cases} \\
& \operatorname{sign}^{-}(\xi)=1-\operatorname{sign}^{+}(\xi)
\end{aligned}
$$




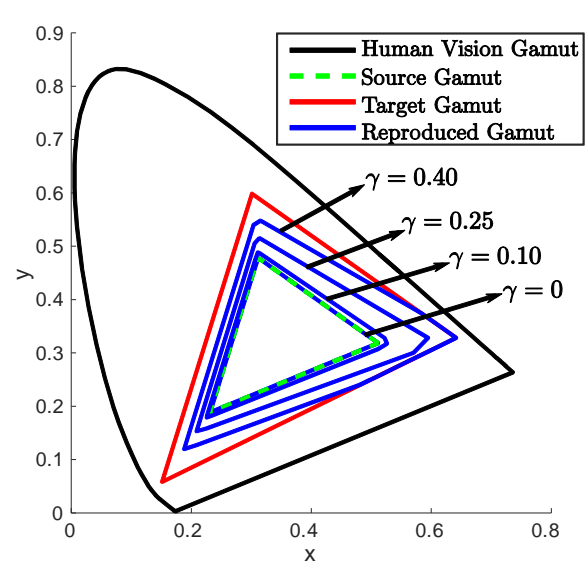

(a)
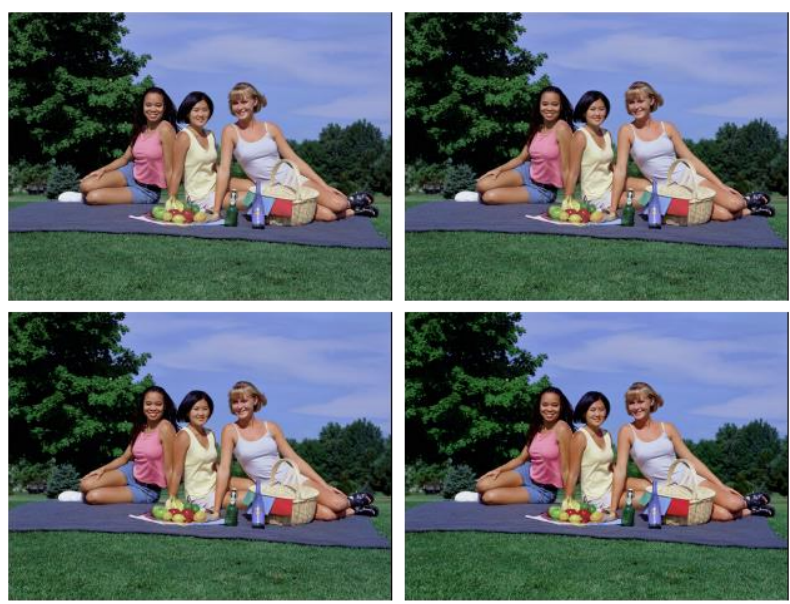

(b)

Figure 3. Gamut extension approach. (a) Gamuts on chromaticity diagram. (b) Gamut extension results. Top left: input image $(\gamma=0)$. Top right: gamut extended image with $\gamma=0.10$. Bottom left: gamut extended image with $\gamma=0.25$. Bottom right: gamut extended image with $\gamma=0.40$. Original image is from the $\mathrm{ClE}^{21}$.

\section{Action of the Proposed GEA on an Image}

In order to perform gamut extension, we first convert the RGB input image to the HSV color space, and then apply Eq. (1) to the saturation component only. The evolution Eq. (1) reaches steady state for each set of values for parameters $\beta, \Delta t$, and $\gamma$, and to show how it modifies the color gamut, an example with several different gamuts on a chromaticity diagram is shown in figure $3 a$ where it can be seen that when $\beta=1, \Delta t=0.10$, and $\gamma=0$ the steady state of the evolution equation produces the original image, and as we increase the value of $\gamma$ the steady state of Eq. (1) yields an image with a larger gamut. Figure $3 \mathrm{a}$ also shows that one can map the colors of an input image to the larger destination gamut just by using a large enough value for $\gamma(\gamma=0.40$ in this case). In order to compute this optimal value for $\gamma$ that allows us to perform GE, we make use of the information provided by the color differences between the source and the destination gamuts (triangle-shaped due to the use of three color primaries):

$$
\gamma=\sqrt[3]{\left|S G_{\text {area }}-D G_{\text {area }}\right|}
$$

where $S G_{\text {area }}$ and $D G_{\text {area }}$ denote the area of the source gamut and the destination gamut, respectively.

For each $\gamma$ value, the corresponding reproduced images are shown in figure $3 \mathrm{~b}$ in which we can notice that the colors of sky, grass and skin have a limited (controlled) increase in saturation, whereas some objects are extended normally such as the bottles, the fruits, the cloths in the basket and the pink shirt. Since we are using a fixed value of $\gamma$ for all the input images with different spatial and chromatic characteristics, our GEA may place a few colors outside the destination gamut that we can map back inside using a gamut reduction algorithm, e.g. the method of Zamir et al. ${ }^{22}$. 


\section{Experiments and Results}

In this section we assess the visual quality of the reproductions produced by the proposed GEA and other methods using the publicly available datasets ${ }^{23,24}$. To compute the results for our method, we work in HSV color space and in Eq. (3) the parameter values that we use are $\beta=1$, $\Delta t=0.10$. Since we are limited by the sRGB standard (that has the same primaries as of BT.709) for the paper, all results presented in this paper are mapped from 'Toy' gamut to sRGB gamut (the primaries of gamuts used in our experiments are mentioned in Table 1). To see the reproductions obtained using our GEA when mapping colors from 'Toy' to DCI-P3 gamut and from BT.709 to DCl-P3, visit the web page: http://ip4ec.upf.edu/gamutmapping.

While the capability of emerging wide-gamut displays in reproducing more vibrant colors helps generating a richer visual experience, it is highly important to render flesh tones in a very careful manner so as to avoid departing away from the content creator's intent. In figure 4 we compare how different GEAs reproduce skin colors, which is always a key issue in movie postproduction. It can be seen in row 2 and row 4 that our GEA applies a limited extension to skin tones but the artificial objects undergo a normal color extension so that the final reproduction appears natural and pleasant, whereas in the same figure it is noticeable that other methods such as same-drive signal (SDS) ${ }^{11}$ and chroma extension ${ }^{11}$ have issues with skin tones and over-saturation. For better comparison, we show in figure 5 the zoomed-in view of regions cropped from figure 4 . The lightness chroma adaptive (LCA) algorithm ${ }^{11}$, due to its inherent behavior of modifying lightness and chroma, produces images with artifacts and the over-enhancement of contrast makes a few colors go towards black (loss of saturation and detail). For example in figure 5, see the napkin in row 2 for artifacts, and the picture of the elephant in the last row depicting some loss of detail. The chroma extension method ${ }^{11}$ shows poor performance when there is a high chromatic object in the scene; one such example is shown in row 4 of figure 5 where it can be seen that the colors of the character are highly saturated.

In general, viewers tend to prefer more saturated colors ${ }^{25}$ when they are not aware of the original colors of either natural or man-made objects, but they are less welcoming to the change in colors of those objects for which they have memory such as shades of sky and grass, etc. Therefore, care should be taken while extending these colors. In figure 4, row 1, we present an example showing that our method performs controlled extension and reproduce the color of the grass accurately, whereas the hybrid color mapping $(\mathrm{HCM})^{11}$, and SDS methods over-saturate the grass region, making it look artificial.

To test the temporal consistency of our method, we apply the proposed GEA on a variety of image sequences $^{23,24}$ with different levels of motion. Representative frames are shown in figure 6 and the complete resultant image sequences with extended gamut are added to the web page for which the link is given above. We confirm that the gamut extended videos obtained using our GEA are free from noticeable spatial and temporal artifacts, hue shifts, flickering and unexpected changes in chroma and brightness of objects. We also want to mention that the quality of the input video is of high importance; if it contains any spatial artifacts due to compression or noise they may become prominent in the reproduced video. 
Table 1. Primaries of gamuts.

\begin{tabular}{|l|c|c|c|c|c|c|}
\hline \multirow{2}{*}{ Gamuts } & \multicolumn{2}{|c|}{ Red Primaries } & \multicolumn{2}{c|}{ Green Primaries } & \multicolumn{2}{c|}{ Blue Primaries } \\
\hline & $\mathrm{x}$ & $\mathrm{y}$ & $\mathrm{x}$ & $\mathrm{y}$ & $\mathrm{x}$ & $\mathrm{y}$ \\
\hline BT.2020 & 0.708 & 0.292 & 0.170 & 0.797 & 0.131 & 0.046 \\
\hline DCI-P3 & 0.680 & 0.320 & 0.265 & 0.690 & 0.150 & 0.060 \\
\hline BT.709/sRGB & 0.640 & 0.330 & 0.300 & 0.600 & 0.150 & 0.060 \\
\hline Toy & 0.570 & 0.320 & 0.300 & 0.530 & 0.190 & 0.130 \\
\hline
\end{tabular}

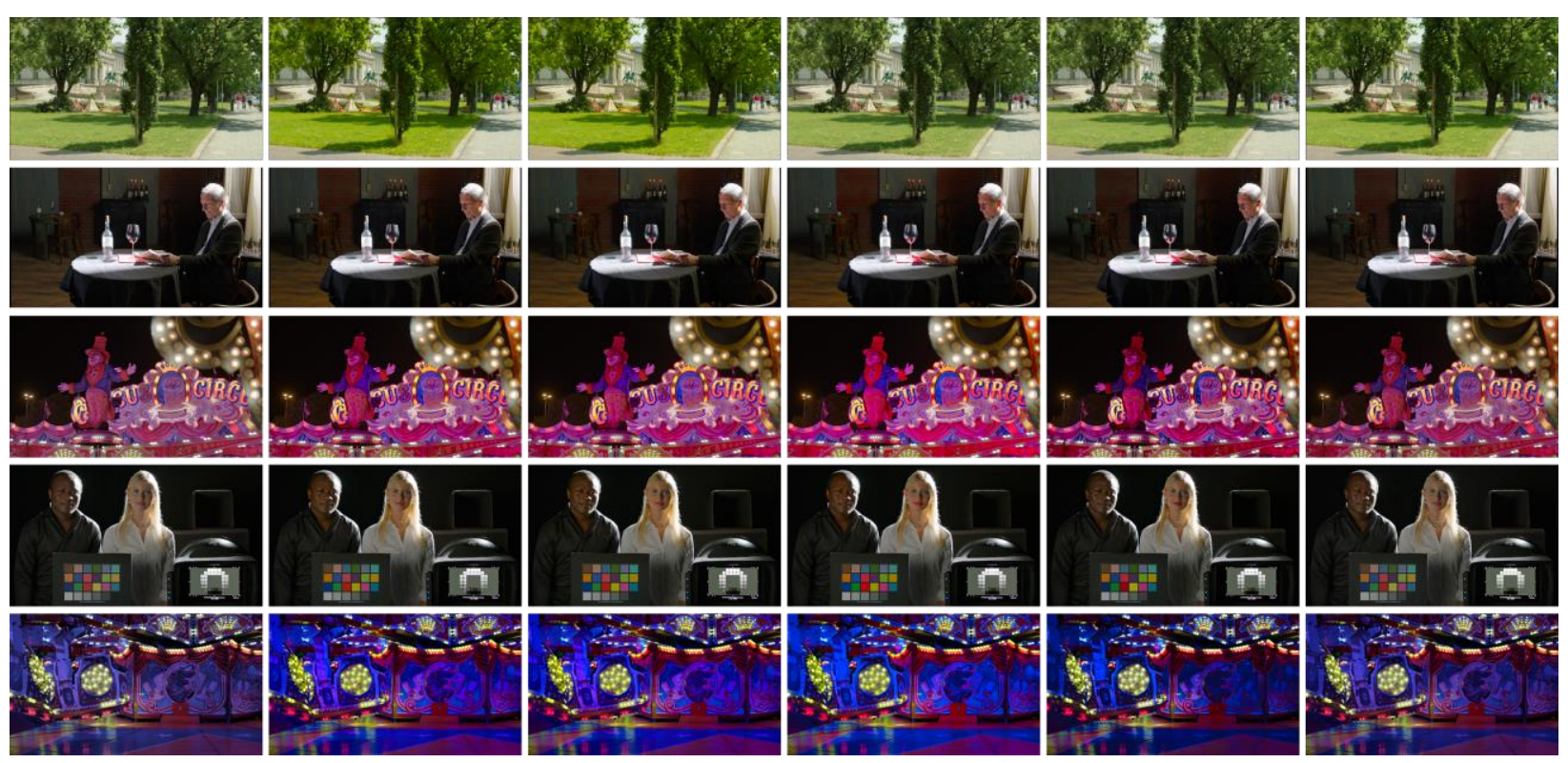

Figure 4. Results: mapping from Toy to sRGB gamut. Column 1: Input image. Column 2: $\mathrm{HCM}^{11}$. Column 3: SDS ${ }^{11}$. Column 4: Chroma extension ${ }^{11}$. Column 5: LCA ${ }^{11}$. Column 6: Our GEA. Original images are from the datasets ${ }^{23,24}$. 

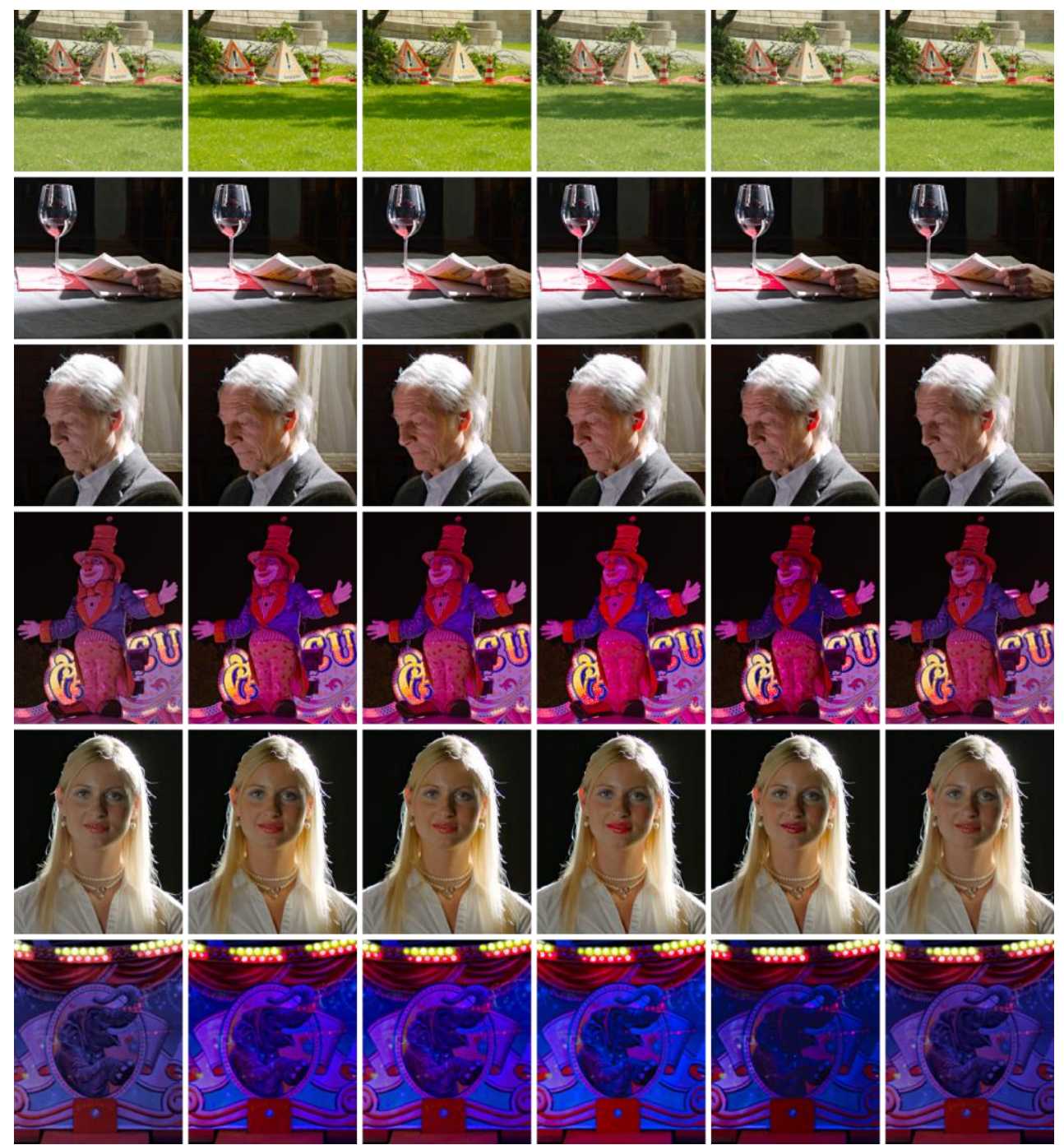

Figure 5. Zoomed-in view of the regions cropped from figure 4. Column 1: Input image. Column 2: $\mathrm{HCM}^{11}$. Column 3: SDS ${ }^{11}$. Column 4: Chroma extension ${ }^{11}$. Column 5: $\mathrm{LCA}^{11}$. Column 6: Our GEA.

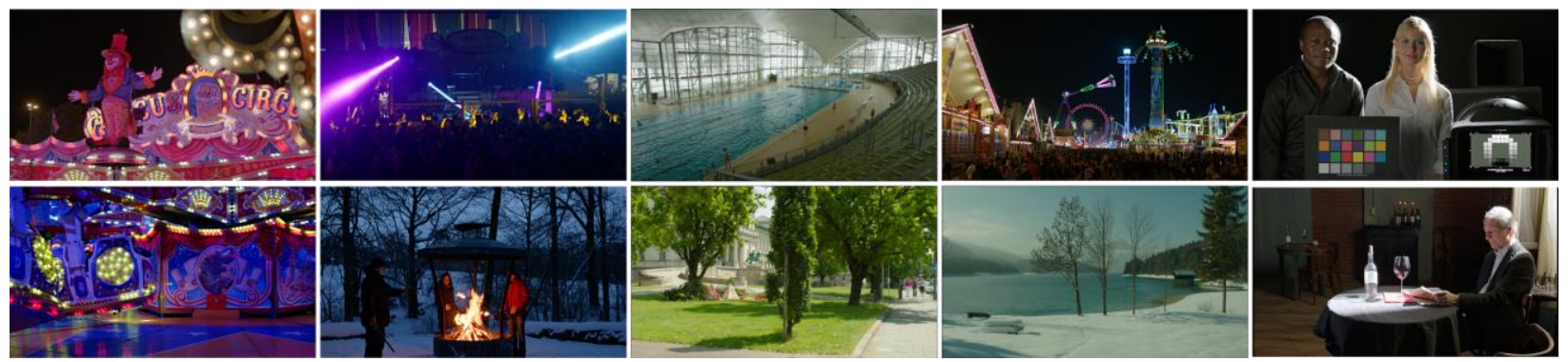

Figure 6. Representative frames of image sequences with sRGB gamut. Original images are from the datasets ${ }^{23,24}$. 


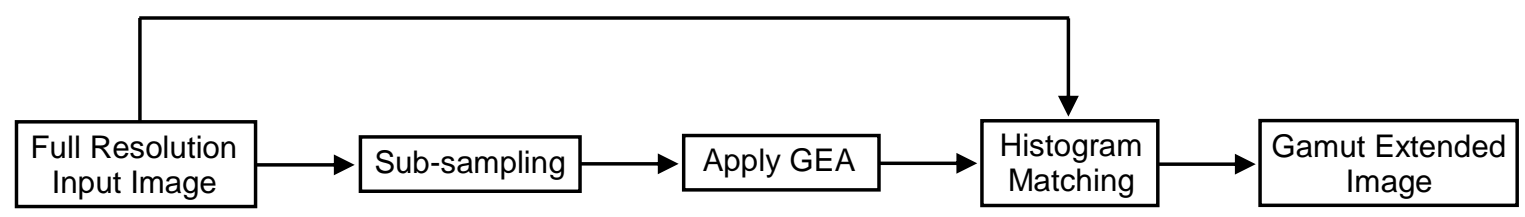

Figure 7. A schematic to reduce the computational cost of the proposed framework.

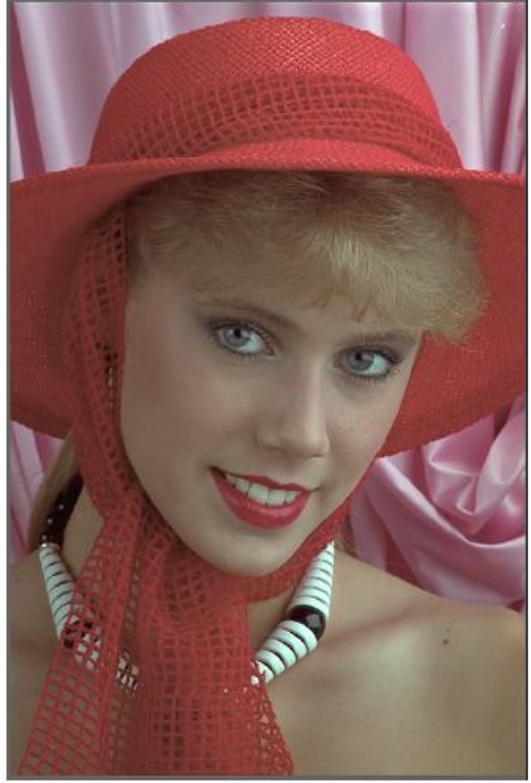

(a)

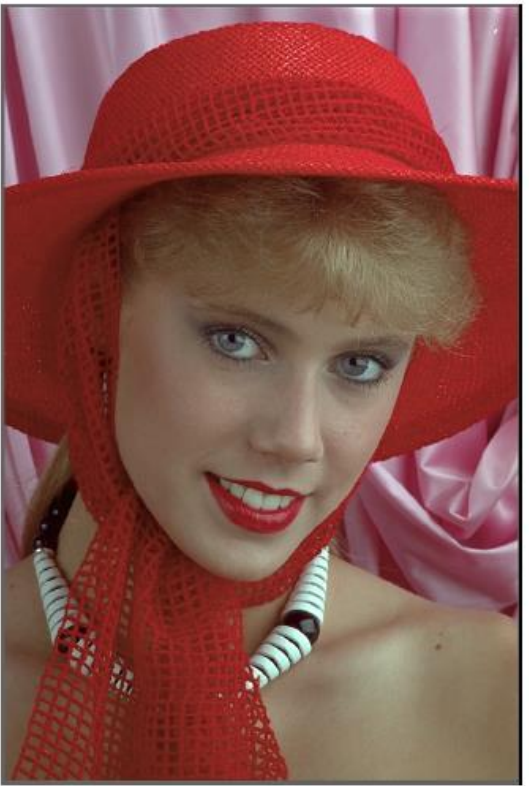

(b)

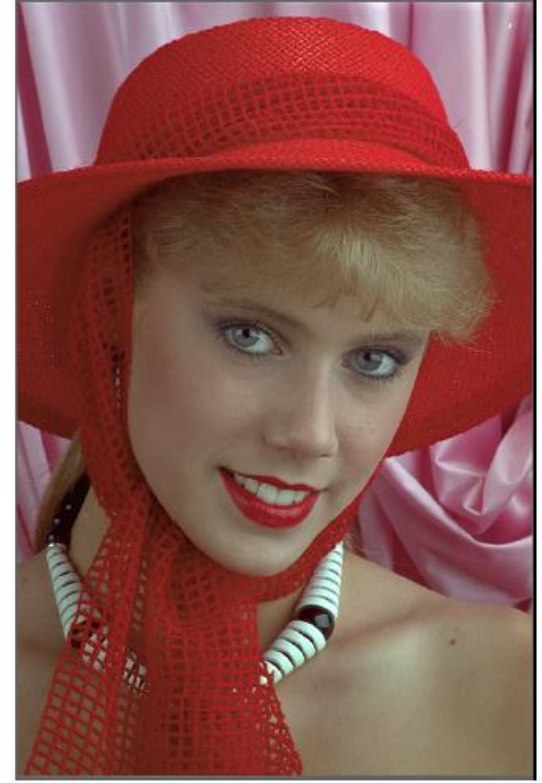

(c)

Figure 8. Example of reducing computational time. (a) Input image. (b) Result of proposed method applied on full resolution image. (c) Result of proposed method applied on sub-sampled image. Original image is courtesy of Kodak.

\section{Making the GEA Faster}

The proposed GEA in a non-optimized MATLAB implementation running on a machine with 8 cores 3.4-GHz CPU takes (on average) 11 seconds to process an image of resolution $656 \times 1080$ pixels. However, the computational cost can be reduced drastically by applying a series of operations (only on the saturation component of an image represented in the HSV color space) that are shown in figure 7 and explained as follows:

- Sub-sampling: the first step is to take the full resolution image and sub-sample it by a scaling factor. The scaling factor of 0.40 provides a good trade-of between the speed and the quality of the reproduction.

- Apply GEA: next apply the proposed GEA on the downsampled image in order to obtain a reduced-size gamut extended image. 
- Histogram Matching: finally we perform histogram matching of the full resolution input image and the subsampled image with extended gamut to obtain the final full resolution gamut extended image.

As shown in figure 8 , using the above mentioned steps we can produce results having the same visual appearance but at a fraction (25\%) of the time.

\section{Conclusion}

In this paper we have presented a GEA that is based on visual perception models and adapts itself according to the content of the input image. On one side, the proposed algorithm modifies less those colors that require a special treatment such as skin tones, less saturated natural objects, neutral colors and some particular memory colors in a controlled manner. On the other side, it extends normally colors of high chromatic (natural and artificial) objects. Our GEA produces gamut extended images and videos that look natural and pleasant in appearance and free from artifacts of any kind. We have also presented a procedure that allows to drastically reduce the computational cost of the proposed GEA while preserving the visual quality of the results.

\section{Acknowledgements}

This work was supported by the European Research Council, Starting Grant ref. 306337, by the Spanish government and FEDER Fund, grant ref. TIN2015-71537-P (MINECO/FEDER,UE), and by the Icrea Academia Award.

\section{Reference}

[1] G. Sharma, Digital Color Imaging Handbook, CRC Press, Inc., 2002.

[2] C. Poynton, J. Stessen, and R. Nijland, "Deploying Wide Color Gamut and High Dynamic Range in HD And UHD", SMPTE Motion Imaging Journal, 124:37-49, 2015.

[3] J. Laird and I. Heynderickx, "Perceptually Optimal Boundaries for Wide Gamut TVs", Proc. of IS\&T/SPIE Electronic Imaging, 2008.

[4] M. R. Pointer, "The Gamut of Real Surface Colours", Color Research \& Application, 5:14555, 1980.

[5] SMPTE RP 431-2:2011, “D-Cinema Quality - Reference Projector And Environment”, 2011.

[6] ITU-R Recommendation BT.709-5, "Parameter Values for the HDTV Standards for Production and International Programme Exchange", 2002.

[7] ITU-R Recommendation BT.2020, "Parameter Values for Ultra High Definition Television Systems for Production and International Programme Exchange", 2012.

[8] B.D. Silverstein, A.F. Kurtz, J.R. Bietry, and G.E. Nothhard, "A Laser-Based Digital Cinema Projector", SID Symposium Digest of Technical Papers, 42:326-329, 2011.

[9] Y. Kusakabe, Y. Iwasaki, and Y. Nishida, "Wide-Color-Gamut Super Hi-Vision Projector", Proc. ITE Annual Convention, 2013 (in Japanese).

[10] http://spectrum.ieee.org/consumer-electronics/audiovideo/lasers-coming-to-a-theater-nearyou. [Online; accessed 02-September-2016]. 
[11] J. Laird, R. Muijs, and J. Kuang, "Development and Evaluation of Gamut Extension Algorithms", Color Research \& Application, 34:443-451, 2009.

[12] H. Pan and S. Daly, "A Gamut-Mapping Algorithm with Separate Skin and Non-Skin Color Preference Controls for Wide-Colorgamut TV", SID Symposium Digest of Technical Papers, 39:1363-1366, 2008.

[13] S. E. Casella, R. L. Heckaman, and M. D. Fairchild, "Mapping Standard Image Content to Wide-Gamut Displays", Proc. Color and Imaging Conference, pp 106-111, 2008.

[14] R. L. Heckaman and J. Sullivan, "Rendering Digital Cinema and Broadcast TV Content to Wide Gamut Display Media", SID Symposium Digest of Technical Papers, 42(1):225-228, 2011.

[15] B. H. Kang, J. Morovic, M. R. Luo, and M. S. Cho, "Gamut Compression and Extension Algorithms Based on Observer Experimental Data", ETRI Journal, 25(3):156-170, 2003.

[16] T. Hoshino, "A Preferred Color Reproduction Method for the HDTV Digital Still Image System", Proc. of IS\&T Symposium on Electronic Photography, pp 27-32, 1991.

[17] H. Anderson, E. Garcia, and M. Gupta, "Gamut Expansion for Video and Image Sets", Proc. International Conference on Image Analysis and Processing Workshops, pp 188-191, 2007.

[18] J. Morovic, Color Gamut Mapping, volume 10. Wiley, 2008.

[19] M. Bertalmío, V. Caselles, and E. Provenzi, "Issues About Retinex Theory and Contrast Enhancement", International Journal of Computer Vision, 83:101-119, 2009.

[20] https://en.wikipedia.org/wiki/Generalised logistic function. [Online; accessed 02-September2016].

[21] CIE, "Guidelines for the Evaluation of Gamut Mapping Algorithms", Technical Report, CIE 156, 2004.

[22] S. W. Zamir, J. Vazquez-Corral, and M. Bertalmío, "Gamut Mapping in Cinematography Through Perceptually-Based Contrast Modification", IEEE Journal of Selected Topics in Signal Processing, 8:490-503, 2014.

[23] S. Andriani, H. Brendel, T. Seybold, and J. Goldstone, "Beyond the Kodak Image Set: a New Reference Set of Color Image Sequences", Proc. IEEE International Conference on Image Processing, pp 2289-2293, 2013.

[24] J. Froehlich, S. Grandinetti, B. Eberhardt, S. Walter, A. Schilling, and H. Brendel, "Creating Cinematic Wide Gamut HDR-Video for the Evaluation of Tone Mapping Operators and HDR-Displays", Proc. of IS\&T/SPIE Electronic Imaging, 2014.

[25] E. A. Fedorovskaya, H. de Ridder, and F. JJ Blommaert, "Chroma Variations and Perceived Quality of Color Images of Natural Scenes", Color Research \& Application, 22:96-110, 1997. 